\title{
Tuning the Bias Sensing Layer: A New Way to Greatly Improve Metal-Oxide Gas Sensors Selectivity
}

\author{
N. Dufour, A. Chapelle, C. Talhi, F. Blanc, B. Franc \\ and $\mathrm{P}$. Menini \\ LAAS-CNRS \\ Toulouse, France \\ ndufour@laas.fr, menini@laas.fr
}

\author{
K. Aguir \\ IM2NP-CNRS \\ Marseille, France
}

\begin{abstract}
The effects of bias current in the sensing layer of resistive Metal-Oxide (MOX) sensors toward gases have been investigated. The behavior of a $\mathrm{WO}_{3}$ thin film deposited by sputtering has been studied. In a first time, while the working temperature is kept constant, it has been found that tuning the polarization of the MOX layer induces changes on its sensitivity. Besides, the behavior of sensitivity versus bias current depends on the nature of the gas. For example, the response to $\mathrm{NH}_{3}$ $10 \mathrm{ppm}$ hugely increases with the bias rise; at the opposite, the polarization has no effect on $\mathrm{NO}_{2}-10 \mathrm{ppm}$ responses. In a second time, the effect of the working temperature changing added to the polarization has been studied. Thus, an optimized temperature-modulated profile has been established and it has been associated with a multivariate analysis in order to quickly and easily discriminate several gases and mixtures. It has been shown this method allows discriminating two gases $\left(\mathrm{NH}_{3}\right.$ and $\mathrm{NO}_{2}$ ) at two concentrations (5 and $10 \mathrm{ppm}$ ) alone and mixed. These results open a new way to increase the selectivity of MOX sensors.
\end{abstract}

Keywords : Gas Sensor, Metal Oxide, Temperature-modulated, Bias-modulated

\section{INTRODUCTION}

Metal oxide thin film sensors have been widely used for gas sensing applications thanks to their sensitivity toward a large variety of gases [1]. The main reaction occurring at the MOX thin film surface is the ionization of adsorbed oxygen, creating a depletion layer at the grain boundaries [2, 3] (Fig.1). The sensing mechanism is based on the reaction between the gas and the Schottky barriers, thus changing the resistivity of the thin film [4]. Considering all the rectifying junctions, the current/voltage characteristic of MOX films exhibits a nonlinear behavior, meaning that their resistance depends on the polarization [5, 6]. Sensitivity toward gases, which is closely related to these phenomena, also turns out to be polarization dependant [7]. The influence of the sensitive layer biasing on sensitivity to carbon monoxide has been observed for a couple of devices yet $[8,9,10]$, underlining distinct behaviors for different metal oxides. However, polarization of MOX layers still suffers from a lack of investigations even though it seems to be of great interest in order to enhance gas sensor performances.

Moreover, this phenomenon can be easily coupled with a well-known method used to increase the gas selectivity, which is the temperature modulation of the sensor, realized in changing the power consumption applied to the heater resistor $[11,12]$.

The work presented here aims to characterize the influence of these parameters so as to improve both the sensitivity and selectivity of MOX sensors.

\section{THEORICAL}

Between 100 and $500^{\circ} \mathrm{C}$, the interaction with oxygen involves its ionosorption in atomic and molecular form [3]. The oxygen chemisorption equation is the following:

$$
(\beta / 2) O_{2}^{g a s}+\alpha \cdot e^{-}+S \leftrightarrow O_{\beta S}^{-\alpha},
$$

where $\mathrm{O}_{2}^{\text {gas }}$ is an oxygen molecule, $e^{-}$is an electron, $S$ is an unoccupied chemisorption site, $\mathrm{O}_{\beta \mathrm{S}}^{-\alpha}$ is a chemisorbed oxygen species, and $\alpha$ and $\beta$ are equal to 1 or 2 for singly or doubly ionised form and for atomic or molecular form respectively.

The nanoparticle-made MOX thin films model consists of strings of grains, where the conduction is limited by the grain boundary voltage barriers [2]. The conductance can thus be expressed as:

$$
G=g q \mu_{S} N_{d} \exp \left(-q V_{s} / k T\right)
$$

where $G$ is the conductance, $g$ is a constant determined by the geometry, $q$ is the elementary charge, $\mu_{s}$ is the electron mobility, $N_{d}$ is the density of donors and $V_{s}$ the potential barrier.

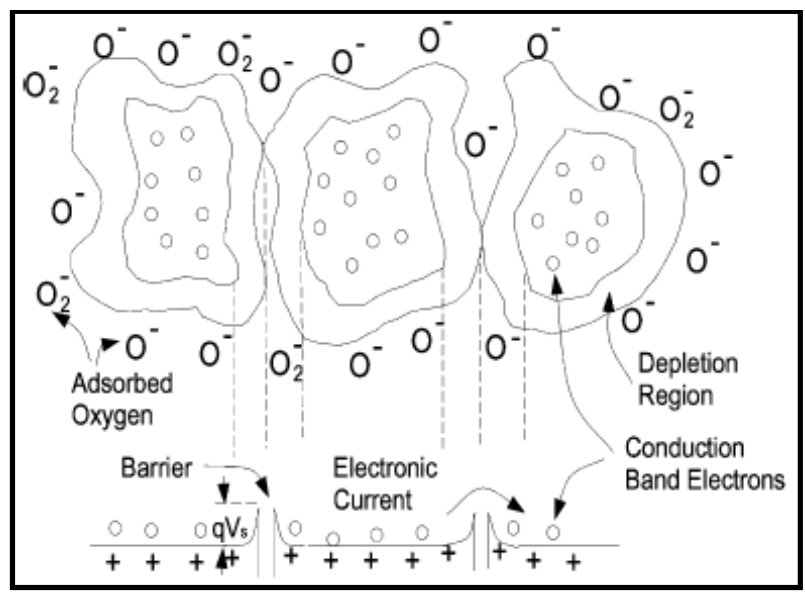

Figure 1. Model of grain boundary of a MOX thin film 
The density of surface states depends on the polarization as described by A. Varpula \& al. [7]; the developed model is grounded on the energy band theory. The reaction between a gas and the ionized MOX layer is related to the surface state occupancy. The variation of the potential barrier height occurring during the reaction is determined by the previous phenomenon. According to (2), the conductance varies with Vs, so the sensitivity is directly linked to the sensing layer polarization.

\section{EXPERIMENTAL}

\section{A. Devices}

The tested devices have been developed on an optimized microhotplate that can work at high temperature and low power consumption $\left(500^{\circ} \mathrm{C}, 55 \mathrm{~mW}\right)$ (Fig. 2$)$.

The platform consists of a silicon bulk on which a thermally resistive bilayer $\mathrm{SiO}_{2} / \mathrm{SiN}_{\mathrm{x}}$ membrane was grown. Afterwards, Ti/Pt metallization is realized by lift-off to define a heating resistor. Contacts were opened in a previously deposited PECVD $\mathrm{SiO}_{2}$ passivation layer. Then, the electrodes of a sensing resistor are defined with another $\mathrm{Ti} / \mathrm{Pt}$ metallization. Finally, the rear side of the bulk was etched to release the membrane in order to increase the thermal resistance and then to limit thermal dissipation. It is then possible to deposit a metal-oxide layer to form the sensing thin film.

The sensitive layer is a thin film of tungsten trioxide $\left(\mathrm{WO}_{3}\right)$ deposited by R.F. reactive magnetron sputtering (RMS) [13].

The post-process annealing of the $\mathrm{WO}_{3}$ has been realized at a temperature of $550^{\circ} \mathrm{C}$ and the maximal operating temperature has been fixed to $500^{\circ} \mathrm{C}$.

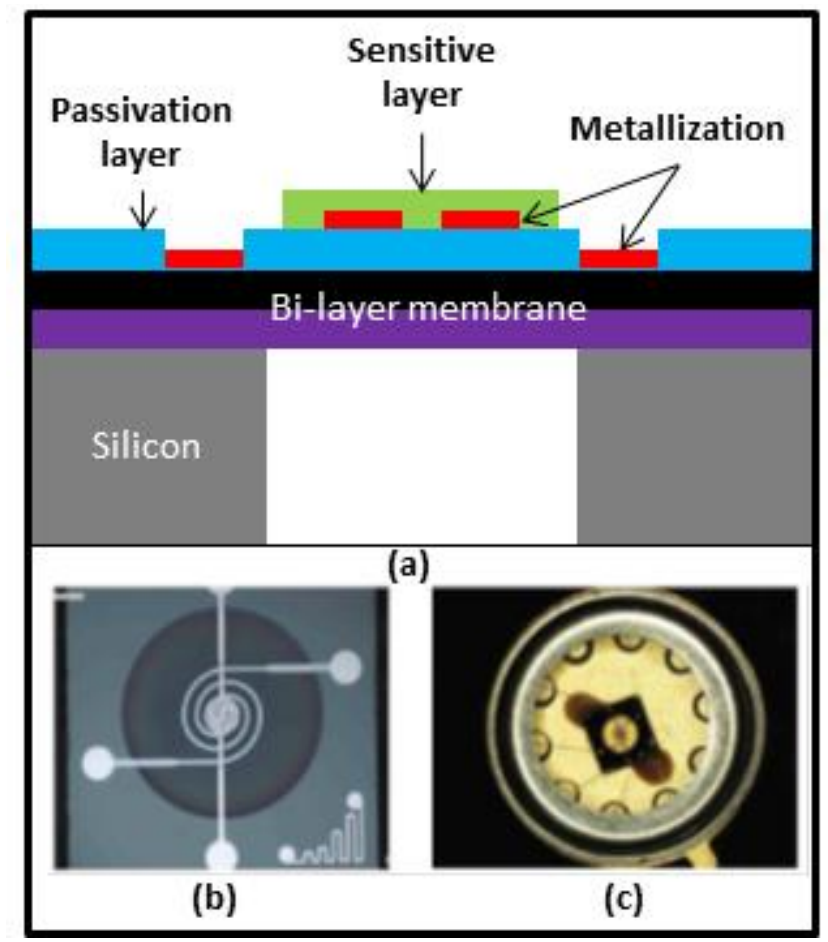

Figure 2. Micro-hotplate gas sensor: (a) cross sectional view, (b) chip top view, (c) chip packaged on a TO-5 support

\section{B. Test Bench}

The sensors are placed into a chamber where gas is flowing (Fig. 3). The composition and relative humidity level of the gas mixture are controlled by Mass Flow Controllers (MFC) and the global constant flow rate $(200 \mathrm{~mL} / \mathrm{min})$ is checked with a debimeter at the chamber outlet. The heating and the sensing resistors are connected to two Source Measurement Units (SMU) Keithley 2400. The whole test bench is automatically controllable thanks to a suitable interface and a dedicated software (CVI LabWindows ${ }^{\circledR}$ ).

\section{Experimental Protocol}

Two elements have been defined to realize different measurements: the way to supply and measure both resistors with the instrumentation, and different polarization profiles applied to them.

\section{1) Source Measurement Units Configurations}

A bias voltage is applied to the heater resistor using one SMU, and the current is measured with the same one. The power consumption can be calculated and the value corresponds to a temperature, thanks to a previous calibration with IR camera.

As for the sensing layer, the most classical method of measurement consists in applying a bias voltage while the current is measured. Both this technique and the dual one (applied current and measured voltage) have been tested and compared. No matter how the polarization is applied, no significant difference has been found in the behavior of sensitivity versus the polarization power as it is shown in Fig.4, thus the measurements were done with a bias current so as to address embedded applications. Two measurement protocols were then considered.

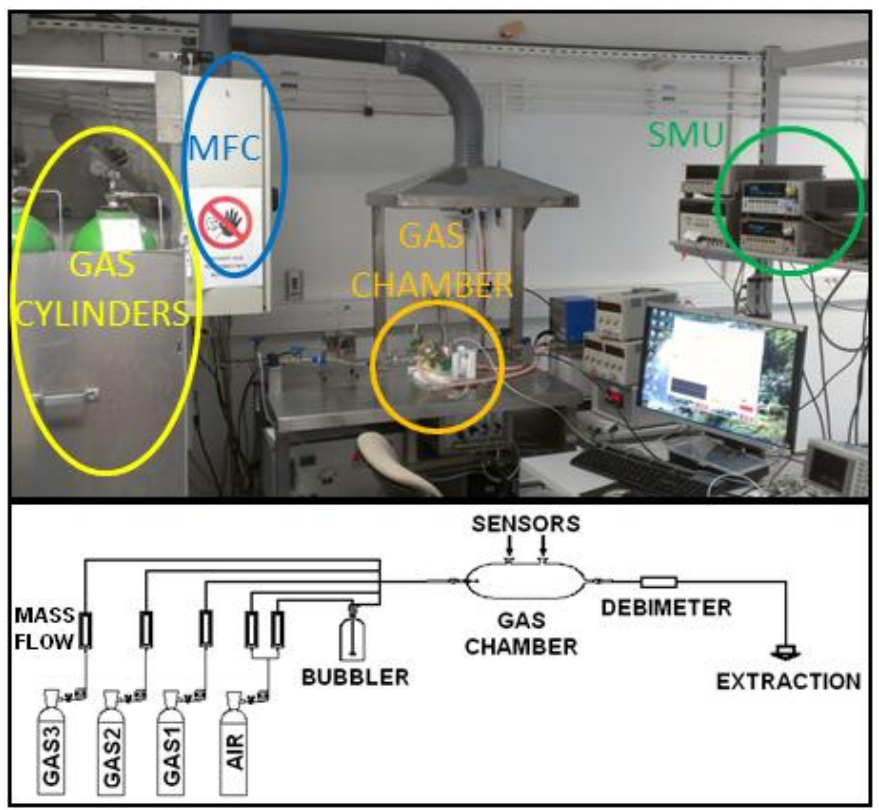

Figure 3. Photo and scheme of the test bench 


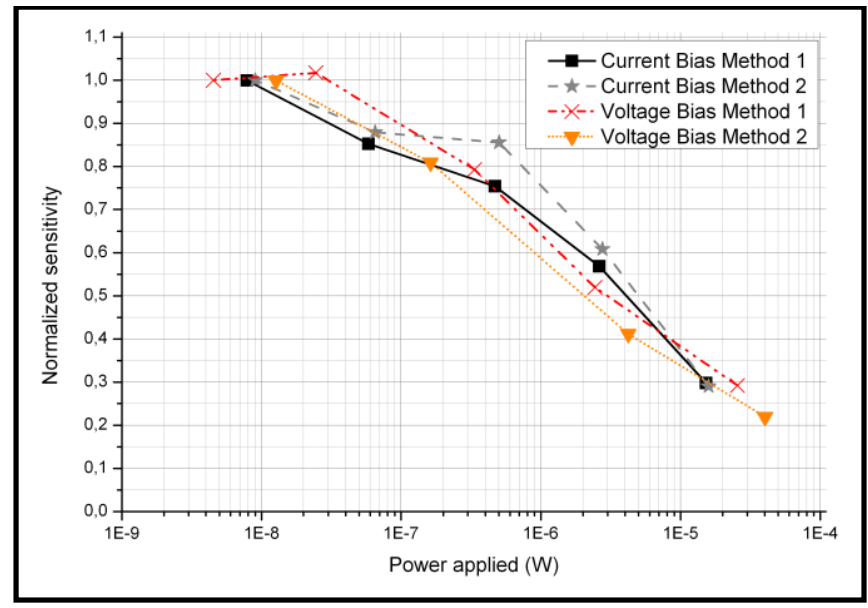

Figure 4. Normalized sensitivities to $\mathrm{NO}_{2} 10 \mathrm{ppm}$ with $\mathrm{WO}_{3}$ sensing layer for different polarization methods

The first one consists in realizing the reaction with gas for each bias current value. After a period of stabilization under dry air (at least one hour), the specific gas is added during a given time while ensuring the global flow remains constant. Finally, the aforementioned gas injection is stopped to let the sensor recover under dry air.

For the second method, the sensor is stabilized under dry air at a particular bias current and then a sweep is realized to cover the desired range of polarization (waiting a few minutes for stabilization at each bias current value). Only then the gas is injected, and after stabilization the same bias current sweep as before is operated, to obtain the values of resistance under gas. The two protocols lead to the same sensitivities, so the second one which allows faster measurement sessions is preferred.

\section{2) Constant Temperature Profile}

The heating resistor biasing voltage is set prior to the test and kept constant afterwards, ensuring a constant working temperature.

In the case of the first tests at constant temperature, the highest operating temperature has been applied $\left(500^{\circ} \mathrm{C}\right)$. Responses of the $\mathrm{WO}_{3}$ layer to three gases (CO-200ppm, $\mathrm{NO}_{2^{-}}$ $10 \mathrm{ppm}$, and $\mathrm{NH}_{3}-10 \mathrm{ppm}$ ) were characterized at different bias currents on the sensing layer, ranging from 0.1 to $10 \mu \mathrm{A}$ (very low dissipation level), and the corresponding sensitivities were compared.

Following this, others lower operating temperatures were performed under the same conditions and the obtained results were compared with the first one.

\section{3) Temperature-modulated Profile}

After testing sensors with stabilized temperatures, a profile with fast temperature changes and different bias currents has been elaborated for the $\mathrm{WO}_{3}$-sensor. Several profiles have been observed, and one that allows good reproducibility, the fastest stabilization and the best discrimination was selected (Fig. 5). The temperature profile is the following: each step lasts 2 seconds; the temperature baseline is the highest operating temperature $\left(500^{\circ} \mathrm{C}\right.$ in this case $)$; the others steps are at lower temperatures $\left(400-300-200^{\circ} \mathrm{C}\right)$; a complete cycle lasts 12 seconds.

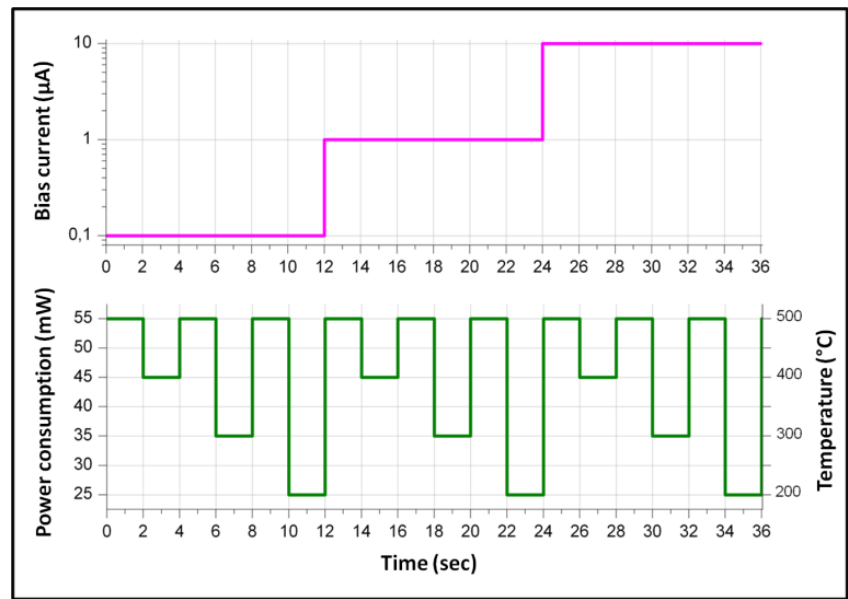

Figure 5. Power comsumption and bias current profiles applied to the $\mathrm{WO}_{3}$ sensor

During one temperature cycle, one bias current is applied to the sensing resistor; three different polarizations are successively set (0.1-1-10 $\mu \mathrm{A}$ in this example); a complete cycle lasts 36 seconds. Both profiles are repeated throughout the test, under various gaseous atmospheres.

\section{Results and Discussion}

The sensitivity (at a given concentration) is calculated as the ratio of the absolute difference between the stabilized resistances of the device under dry air and under the specific gas to the resistance under dry air:

$$
S=\left(\left|R_{\text {air }}-R_{\text {gas }}\right| / R_{\text {air }}\right) \times 100 .
$$

It is defined for each bias current and then normalized in order to emphasize the general trend of the variation of sensitivity with polarization and to make the results for different gases comparable. The lowest bias current value, namely $100 \mathrm{nA}$, has been chosen as the reference for the normalization and the corresponding sensitivity is then set to +1 or -1 , according to the following formula:

$$
S_{i} \text { norm }= \pm\left[1-\left(\left(S_{100 n A}-S_{i}\right) / S_{100 n A}\right)\right] .
$$

The sign plus is used for an oxidizing type reaction, meaning the response mechanism results in an increase of the resistance, for n-type materials as $\mathrm{WO}_{3}$, whereas the sign minus corresponds to a reducing type reaction. This shaping enables the comparison of the sensitivity evolution with increasing bias currents for all the available gases.

\section{1) Maximal Operating Temperature}

The responses of the $\mathrm{WO}_{3}$ layer to the three gases $(\mathrm{CO}$, $\mathrm{NO}_{2}$ and $\mathrm{NH}_{3}$ ) were recorded and the corresponding sensitivities were calculated using (3) and compared for three different bias currents: $0.1,1$ and $10 \mu \mathrm{A}$ (Fig.6).

The behavior of the sensor to CO-200ppm changes significantly with the polarization value, since the response has an oxidizing type for the lowest bias currents and a reducing type for the highest one.

Unlike the $\mathrm{CO}$ response, the $\mathrm{NO}_{2}$ response is kept in the same type (oxidizing) at each bias value. The sensitivity to this gas was around $200 \%$. 


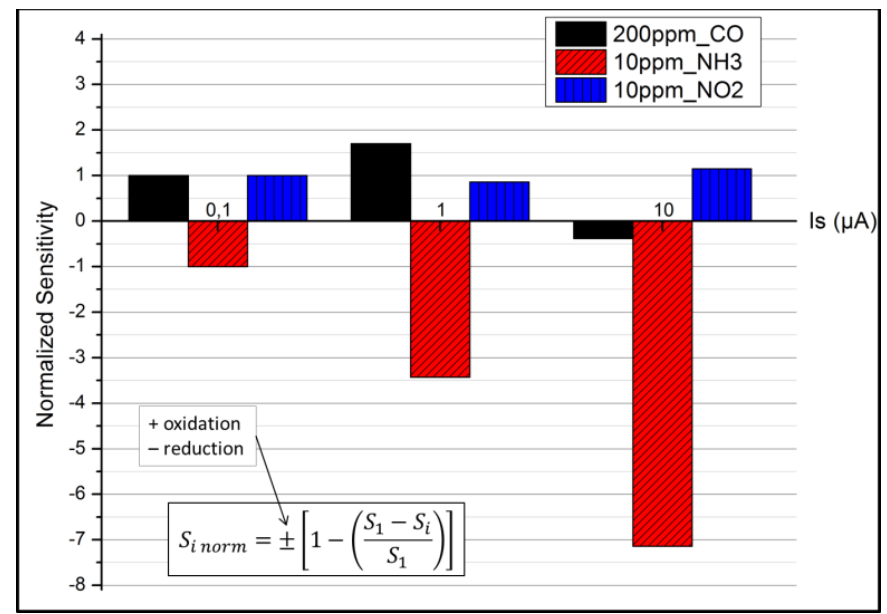

Figure 6. Normalized sensitivities of $\mathrm{WO}_{3}$ sensitive layer to three different gases at $500^{\circ} \mathrm{C}$

Its response to $\mathrm{NH}_{3}$ underlines a huge increase of sensitivity with stronger bias currents (from $3.5 \%$ at $0.1 \mu \mathrm{A}$ to $25 \%$ at $10 \mu \mathrm{A})$.

So, at one operating temperature and for three different gases and three different bias current, the behavior of the sensor is totally different. Such a change turns out to be of primary importance as far as the tuning of selectivity is concerned.

\section{2) Others Constant Temperatures}

After testing the sensor at the highest operating temperature, some measurements at a lower temperature have been realized, in order to compare the influence of this other parameter.

The $\mathrm{WO}_{3}$ sensor has been tested at $200^{\circ} \mathrm{C}$ and the obtained results were compared to those at $300^{\circ} \mathrm{C}$. The most notable changes were observed under $\mathrm{NH}_{3}-10 \mathrm{ppm}$ (Fig. 7).

In this case, the response from this sensor to this gas was more than doubled between $100 \mathrm{nA}$ and $10 \mu \mathrm{A}$ (from 15 to $37 \%$ ) at $300^{\circ} \mathrm{C}$, whereas it was divided by two between the same bias currents (from 28 to $16 \%$ ) at $200^{\circ} \mathrm{C}$.

Based on this observation, it has been decided to inspect the bias influence with a temperature-modulated profile.

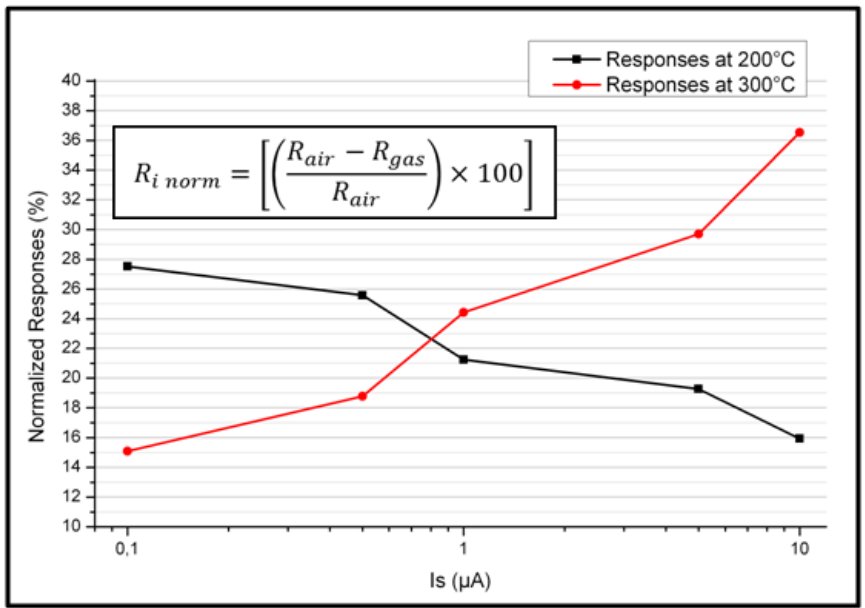

Figure 7. Normalized responses of $\mathrm{WO}_{3}$ sensitive layer to $\mathrm{NH}_{3} 10 \mathrm{ppm}$ for two different heater constant temperatures

\section{3) Temperature-modulated}

The optimized temperature-modulated profile presented in a previous paragraph (Fig. 5) has been applied to the $\mathrm{WO}_{3}$ sensor. It has been repeated during the whole test. Two different gases at two different concentrations have been injected: $\mathrm{NO}_{2}$ and $\mathrm{NH}_{3}$ at 5 and 10ppm. A gas profile has been imagined (Fig. 8). After a stabilization period (around one hour and a half), each gas is injected individually in a first time, and then both gases are injected together at the different concentrations. One gas injection lasts twelve minutes and two air sequences are interposed between each one.

A principal component analysis (PCA) has been realized on the data (Fig. 9). The presented results show a perfect discrimination between each gas and each mixture for all concentrations, thanks to the combination of temperaturemodulated profile and bias modulation. Moreover, the displacement of the points in function of the gaseous ambiance follows two vectors generated by the pure gases. The origin of these vectors is the air and they follow the increase of the concentration.

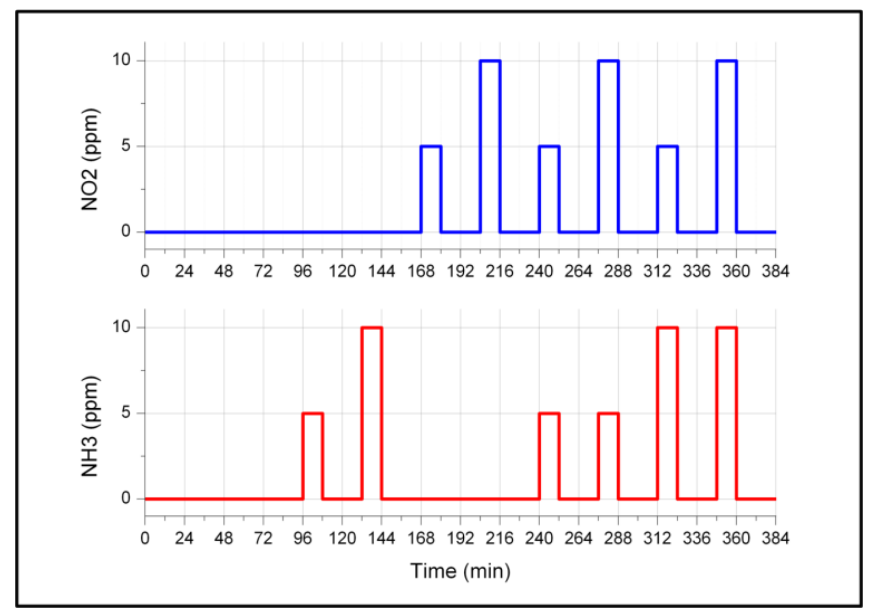

Figure 8. Gas profile applied to the $\mathrm{WO}_{3}$ sensitive layer with the temperature-modulated profile

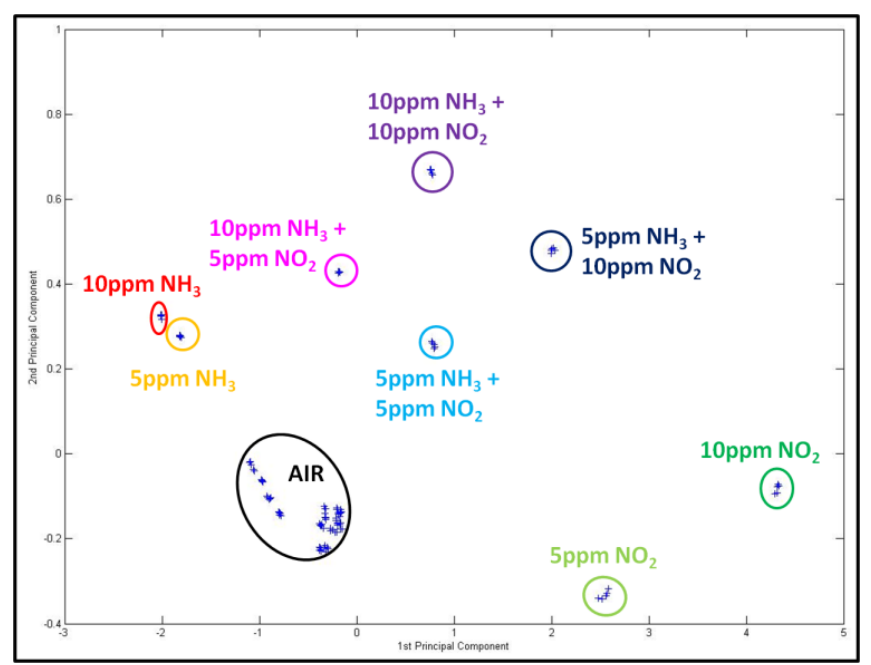

Figure 9. Principal component analysis of $\mathrm{WO}_{3}$ sensitive layer 


\section{CONCLUSION}

In most cases, the sensing layer polarization is an intrinsic parameter, due to the association with others resistor, as in a divider bridge for example. In our study, we have decided to control it directly with instrumentation, and observe the effect of changing this value under different gases. $\mathrm{WO}_{3}$ has been first chosen as sensing material.

Polarization of the sensing layer has been inspected in different conditions: first, at constant temperature, it has been shown a highly dependent response to gases according to the polarization. Then, as for the temperature impact, the $\mathrm{WO}_{3}$ sensing layer has been tested under $\mathrm{NH}_{3}-10 \mathrm{ppm}$ with two different operating temperatures $\left(200\right.$ and $\left.300^{\circ} \mathrm{C}\right)$, revealing opposite behaviors. Indeed, at the lowest temperature, the sensitivity decreases strongly when the bias current is increased, and conversely for the highest one. And finally, a temperature-modulated profile has been applied to the sensor under two gases and two concentrations. A multivariate analysis (PCA) allows discrimination of each gas (pure and mixture) with clear distribution according to concentration.

Combining the effects of the sense and the heater polarizations, which are post-process tunable parameters, enables to modulate the sensitivity of each sensor to each specific gas. That provides many possibilities to enhance the selectivity; a highly discriminating multi-sensor could then be realized.

\section{ACKNOWLEDGMENT}

The authors express their gratitude to RENAULT S.A.S. for the financial support.

\section{REFERENCES}

[1] C. Wang, L. Yin, L. Zhang, D. Xiang and R. Gao, "Metal oxide gas sensors: sensitivity and influencing factors," Sensors, vol. 10, pp. 20882106,2010
[2] J. Ding, T.J. McAvoy, R.E. Cavicchi and S. Semancik, "Surface state trapping models for $\mathrm{SnO}_{2}$-based microhotplate sensor," Sensors And Actuators B, vol. 77, pp. 597-613, 2001.

[3] N. Barsan and U. Weimar, "Conduction model of metal oxide gas sensors," Journal of Electroceramics, vol. 7, pp. 143-167, 2001.

[4] N. Barsan and U. Weimar, "Understanding the fundamental principles of metal oxide based gas sensors; the example of $\mathrm{CO}$ sensing with $\mathrm{SnO} 2$ sensors in the presence of humidity," Journal of Physics: Condensed Matter, vol. 15, pp. 813-839, 2003.

[5] Y. Min, "Properties and sensor performance of zinc oxide thin films," $\mathrm{PhD}$ in Electronic, Photonic and Magnetic Materials, Massachusetts Institute Of Technology, September 2003.

[6] V.V. Simakov, O.V. Yakusheva, A.I. Grebennikov and V.V. Kisin, "Current-voltage characteristics of thin-film gas sensor structures based on tin dioxide," Technical Physics Letters, vol. 31, pp. 339-340, 2005.

[7] A. Varpula, S. Novikov, J. Sinkkonen and M. Utriainen, "Bias dependent sensitivity in metal-oxide gas sensors," Sensors And Actuators B, vol. 131, pp. 134-142, 2008.

[8] S.M.A. Durrani, "Biasing voltage dependence of sensitivity of electron beam evaporated $\mathrm{SnO}_{2}$ thin film CO sensor," Sensors, vol. 6, pp. 11531160, 2006.

[9] S.M.A. Durrani and M.F. Al-Kuhaili, "Effect of biasing voltages and electrode metals and materials on the sensitivity of electron beam evaporated $\mathrm{HfO}_{2}$ thin film $\mathrm{CO}$ sensor," Materials Chemistry and Physics, vol. 109, pp. 56-60, 2008.

[10] S.M.A. Durrani, M.F. Al-Kuhaili, I.A. Bakhtiari and M.B. Haider, "Investigation of the carbon monoxide gas sensing characteristics of tin oxide mixed cerium oxide thin films," Sensors, vol. 12, pp. 2598-2609, 2012.

[11] A. Burresi \& al., "Dynamic, CO recognition in presence of interfering gases by using one MOX sensor and a selected temperature profile", Sensors and Actuators B, vol. 106, pp. 40-43, 2005.

[12] F. Parret \& al., "Improvement of micromachined $\mathrm{SnO}_{2}$ gas sensors selectivity by optimised dynamic temperature operating mode", Sensors and Actuators B, vol.118, pp.276-282, 2006.

[13] C. Lemire \& al., "Reactive R.F. magnetron sputtering deposition of $\mathrm{WO}_{3}$ thin films", Sensors and Actuators B, vol.84, pp.43-48, 2002. 